Article

\title{
Fires Monitoring by means of Spectral Indices from Sentinel-2 Data: the Case Study of the Vesuvius and Lattari Mountains, Campania (Italy)
}

\author{
Domenico Antonio Giuseppe Dell'Aglio ${ }^{1} \mathbb{D}$, Carmine Gambardella ${ }^{2} \mathbb{D}$, Massimiliano Gargiulo ${ }^{1}$, \\ Antonio Iodice $^{1}(\mathbb{D})$, Rosaria Parente ${ }^{2} \mathbb{D}$, Daniele Riccio ${ }^{1}\left(\mathbb{D}\right.$, and Giuseppe Ruello ${ }^{1}$ \\ 1 Department of Electrical Engineering and Information Technology (DIETI), University Federico II, \\ 80125 Naples, Italy; massimiliano.gargiulo@unina.it (M.G.); \\ domenicoantoniogiuseppe.dellaglio@unina.it (D.A.G.D.); iodice@unina.it (A.I.); riccio@unina.it (D.R.); \\ ruello@unina.it (G.R.) \\ 2 BENECON S.C.aR.L., UNESCO Chair on Landscape, Cultural Heritage and Territorial Governance, Via I \\ Maggio, 81030, Aversa (CE), Italy; carmine.gambardella@benecon.it (C.G.); rosaria.parente@benecon.it (R.P.) \\ * Correspondence: massimiliano.gargiulo@unina.it; Tel.: +39-081-768-3512
}

\begin{abstract}
Forest fires are part of a set of natural disasters that have always affected regions of the world typically characterized by a tropical climate with long periods of drought. However, due to climate change in recent years, other regions of our planet have also been affected by this phenomenon, never seen before. One of them is certainly the Italian peninsula, and especially the regions of southern Italy. For this reason, the scientific community, as well as remote sensing one, is highly concerned in developing reliable techniques to provide useful support to the competent authorities. In particular, three specific tasks have been carried out in this work: (i) fire risk prevention, (ii) active fire detection, and (iii) post-fire area assessment. To accomplish these analyses, the capability of a set of spectral indices, derived from spaceborne remote sensing (RS) data, is assessed to monitor the forest fires. The spectral indices are obtained from Sentinel-2 multispectral images of the European Space Agency (ESA), which are free of charge and openly accessible. Moreover, the twin Sentinel-2 sensors allow to overcome some restrictions on time delivery and observation repeat time. The performance of the proposed analyses were assessed experimentally to monitor the forest fires occurred in two specific study areas during the summer of 2017: the volcano Vesuvius, near Naples, and the Lattari mountains, near Sorrento (both in Campania, Italy).
\end{abstract}

Keywords: fire monitoring; Sentinel-2; time series; Italy wildfires; active fire detection; pre-fire analysis; burned area mapping

\section{Introduction}

Nowadays the Earth observations are extensively used to manage risk (floods, subsidence, landslide), to monitor land usage, and so forth [3]. The damage caused by fires is a key element both for the environment itself and for humans. In fact, fires are the main cause of forest loss around the world and even animal heritage loss. Therefore, this general impoverishment of the ecosystem corresponds to an increase of the hydro-geological instability (especially in mountainous areas with high slopes), threatening any human settlements downstream and around the burned areas. Although on the one hand, these tragic events have highlighted the limits of the current preventive measures, on the other hand they have determined the need to find alternative ways that can support the existing ones and the relevant authorities.

Therefore, satellite remote sensing can certainly be helpful to support forest fires monitoring activities. It allows some important advantages such as the ability to find information on large areas in relatively quick way (including those inaccessible and/or dangerous to humans). At the moment, fires 
monitoring services based on the processing of satellite data [1,2] have three main aims: (i) fire risk assessment, (ii) active fire detection, and (iii) burned area mapping.

Among these, the first is certainly the most difficult to handle for the remote sensing scientists. In fact, to the best of our knowledge, a specific technique able to well perform this task is still absent. Nowadays, prevention mechanisms are mainly based on the processing of meteorological data and on the use of human resources. Therefore, in order to find a relationship between the fire ignition event, the status of the vegetation and the climatic data, a time series based on Normalized Differential Water Index (NDWI) [3] and Enhanced Vegetation Index (EVI) [4] products, achieved by MODIS data, has been analyzed [5] .

In order to detect active fires, the joint use of Short Wavelength Infra-Red (SWIR) and Near Infra-Red (NIR) spectral bands must be taken into account [6-8]. In particular, the SWIR frequencies are very useful because they are transparent with respect to smoke clouds during the active fires [9].

Eventually, the third objective concerns the post-fire management and, in particular, the evaluation of the burned area extension. In fact, accurate information about the extension of the burned areas is needed to calculate losses both in terms of economic and environmental impacts. Usually a good method to compute this task is based on the so-called change detection analysis $[6,8,10]$. It basically consists in comparing the images of the same scene on the ground but acquired at different times. In

\begin{tabular}{l|c|c}
\hline $\begin{array}{l}\text { Spatial } \\
\text { Resolution }[\mathrm{m}]\end{array}$ & $\begin{array}{c}\text { Bands } \\
\text { (Bands Number) }\end{array}$ & $\begin{array}{c}\text { Wavelength Range } \\
{[\mu \mathrm{m}]}\end{array}$ \\
\hline 10 & $\begin{array}{c}\text { Blue (2), Green (3), Red (4), } \\
\text { and NIR (8) }\end{array}$ & $0.490-0.842$ \\
\hline 20 & $\begin{array}{c}\text { Vegetation Red Edge (5,6,7,8A) } \\
\text { and SWIR (11,12) }\end{array}$ & $0.705-2.190$ \\
\hline 60 & $\begin{array}{c}\text { Coastal Aerosol (1), } \\
\text { Water Vapour (9), and SWIR (10) }\end{array}$ & $0.443-1.375$ \\
\hline
\end{tabular}

Table 1. The 13 Sentinel-2 bands.

order to detect wildfires and monitoring forest areas under fire risk, current remote sensing systems, such as the European Forest Fire Information System (EFFIS) [11] or the Advanced Fire Information System (AFIS) [12], are essentially based on data processing from MODIS [1] and VIIRS [2] satellite sensors. On the one hand, they return multispectral images that have good requirements in terms of frequency of observations (16 days for both sensors). On the other hand, they have a very low spatial resolution on the ground (that is hundreds of meters), resulting in an inability to detect the exact position of active fires and the exact extension of the burned areas. The small fires aftermaths are surely less significant than large fires in terms of climate changes, but play a significant role in the usage of land and in total gas emissions. In order to overcome these bottlenecks, some works have used data from the Landsat and the ESA Sentinel-2A/2B $[13,14]$ satellite sensors. The Landsat bands, from Visible to SWIR, are provided at 30-m spatial resolution whereas the Panchromatic at 15-m. On the contrary these bands from Sentinel-2 (S-2) are acquired at different spatial resolution, from $60-\mathrm{m}$ to $10-\mathrm{m}$, and we can see the spectral response of these bands in Table 1. Moreover, the S-2 data even provide excellent performance in terms of acquisition rate (passages on same area every 5 days through the combined use the twin satellite sensors). As we can see in Table 1, they can acquire data on 13 spectral channels in the Visible, NIR and SWIR bands at different spatial resolutions [13,14]. However, the Sentinel-2 NIR and SWIR are provided at 10-m and 20-m spatial resolution respectively, as reported in Table 1. In order to further improve the spatial resolution and to obtain more accurate active fires mapping, some works resort to super resolution techniques on SWIR bands. Thus, the 10-m spatial resolution for SWIR bands is achieved using data from the S-2 bands at higher resolution $[9,15]$. The rest of the paper is organized as follows: First, in Section 2 we describe the dataset and 


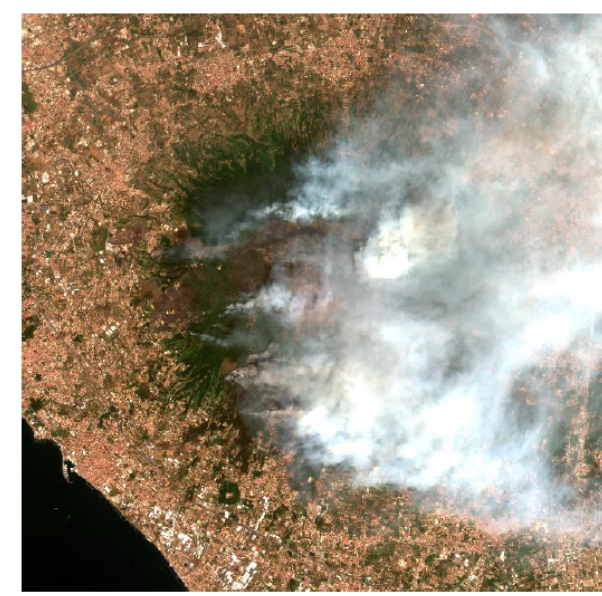

(a)

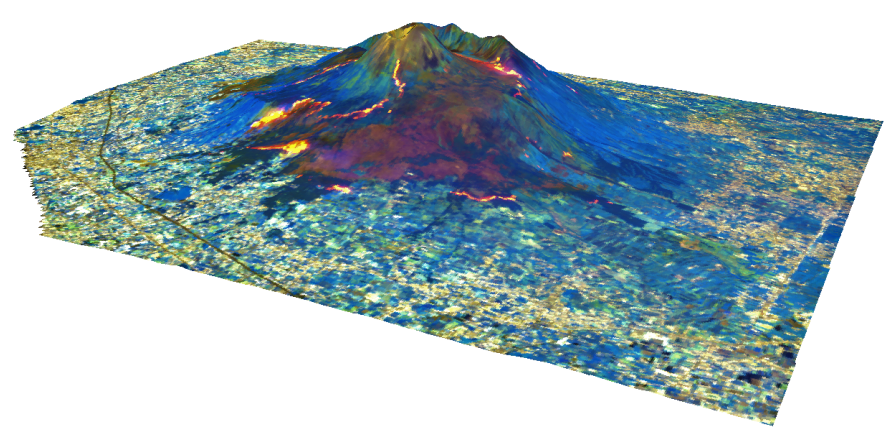

(b)

Figure 1. (a) RGB image of the fire on Vesuvius from Sentinel-2 data acquired on July 12th 2017 and (b) 3-D false colour composite ( $\rho_{12}, \rho_{11}$ and $\rho_{8 A}$ bands) of the same data, obtained using the SRTM DEM.

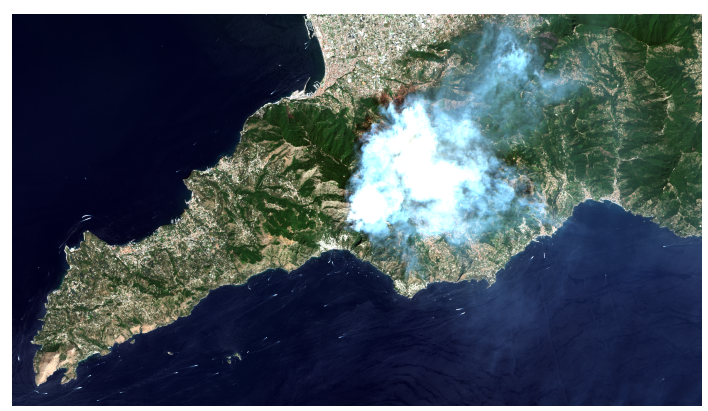

(a)

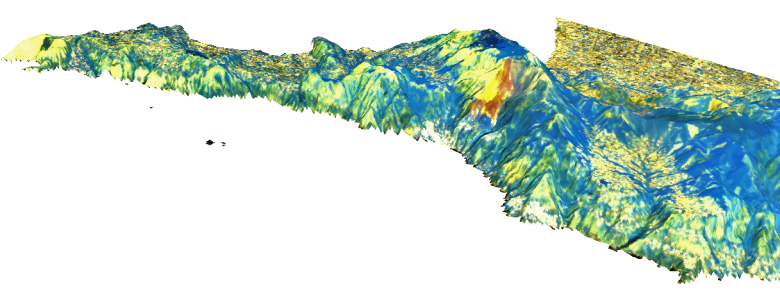

(b)

Figure 2. (a) RGB image of the fire on Lattari mountains from Sentinel-2 data acquired on 12 July 2017 and (b) 3-D false colour composite ( $\rho_{12}, \rho_{11}$ and $\rho_{8 A}$ bands) of the same data, obtained using the SRTM DEM.

the investigated study areas. Then, in Section 3 we focus on the proposed analysis of the three main above-described aims. Thus, in Section 4 we show some experimental results. Eventually, we highlight conclusions and future work.

\section{Study Area and Dataset}

The study of the fire effects are very important, considering that in less than seven months (from winter to summer season of 2017), about 75.000 hectares of woodland were destroyed, and in particular $65 \%$ of the total damages in the Italian regions. For all these analyses, we selected some examples of fires that devastated the Italian country, specifically south Italy. Among these burned areas important forest heritage was also involved, such as those of the volcano Vesuvius and the Lattari mountains, both located in Campania, in the south of Italy (Fig.1 and Fig.2).

For the specific investigated study areas, we used Sentinel-2 standard Level-1C which represent the Top-Of-Atmosphere (TOA) in cartographic geometry (UTM/WGS84 projection). However, in multi-temporal analysis, conducted in this work, we performed the atmospheric correction on Level-1C products using the Sen2Cor Processor to create the Bottom-Of-Atmosphere (BOA) reflectances (i.e. Level-2A products). In Table 2 we can see the whole dataset exploited for the proposed analysis, where the possible presence of the cloud covering is also shown. 


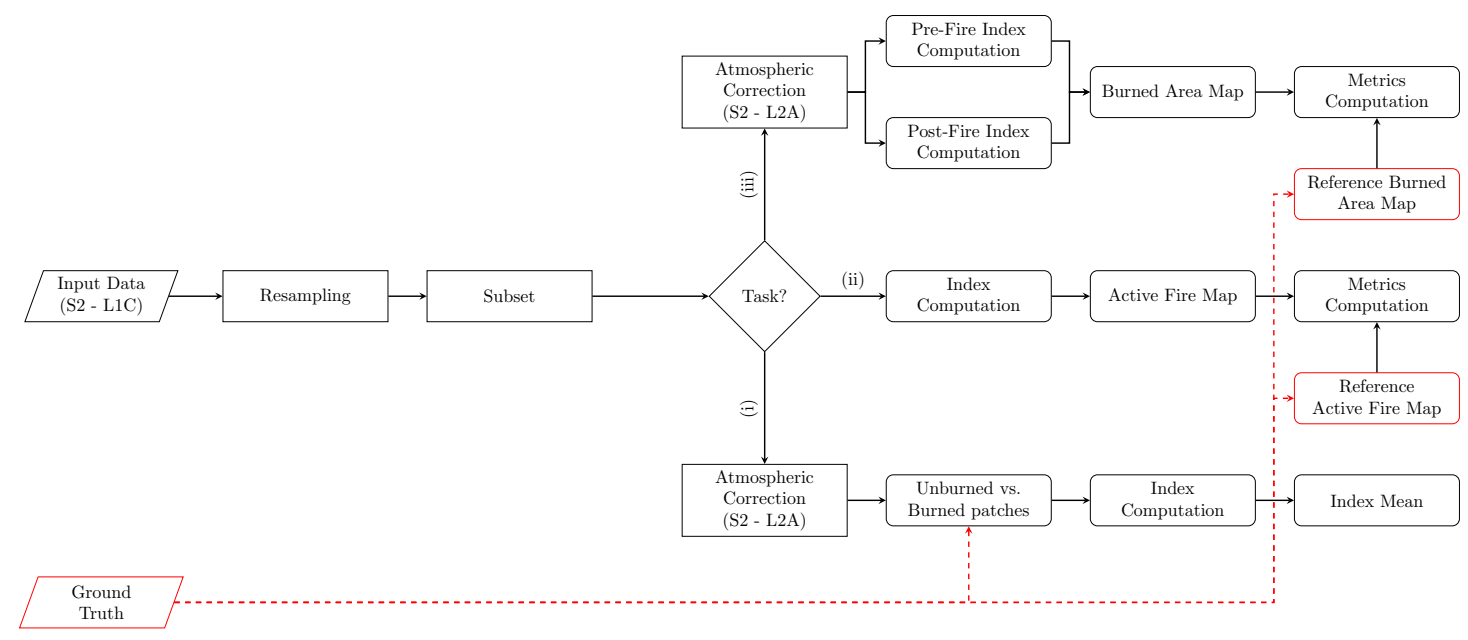

Figure 3. General workflow of the proposed analysis.

\section{Proposed analysis}

In this Section we present the analysis conducted on the aforementioned target sites for the three proposed topics. The general workflow is depicted in Fig.3. The core of this workflow is based on the choice of the task, so after the decision a different branch of the graph must be followed: (i) fire risk prevention, (ii) active fire detection, and (iii) post-fire area assessment. However, in all these analyses we started from the Sentinel-2 Level-1C (S2-L1C), as shown in Figure 3, and we realized a specific resampling taking into account the considered task. Then, we focused on the study area. In the next sections, we analyse in more details the different branches.

\subsection{Pre-fire analysis}

In order to find a relationship between the fire ignition event and the status of the vegetation, the pre-fire analysis is conducted on a time series based on Normalized Differential Water Index (NDWI) [3]. The NDWI is defined as [3]:

$$
N D W I=\frac{\rho_{N I R}-\rho_{S W I R}}{\rho_{N I R}+\rho_{S W I R}}
$$

where $\rho_{\text {NIR }}$ and $\rho_{S W I R}$ are the BOA reflectance values of the NIR and SWIR band, respectively. The NDWI, obtained from freely-available Sentinel-2 Level-2A (S2 - L2A) data, is calculated as:

$$
N D W I=\frac{\rho_{8}-\rho_{11}}{\rho_{8}+\rho_{11}}
$$

where $\rho_{8}$ is the NIR band, centered at the wavelength of $842 \mathrm{~nm}$; while $\rho_{11}$ is the SWIR band, centered at $1610 \mathrm{~nm}$. The Level-2A products provide atmospheric corrected reflectance images which are required when we want to compare spectral index values of multiple dates [16]. Because of its main characteristic to monitor changes in water content of leaves [3], the NDWI has been used to exploit a time series analysis from Sentinel-2 Level-2A data covering almost one year of acquisitions (from December 2016 to December 2017, listed in Table 2).

\subsection{During-fire analysis}

For the during-fire task some Active Fire Indices (AFIs), mainly used to this aim in literature due to their computational simplicity and their physical properties $[7,8,10,17]$, are compared. The AFIs are derived through a proper combination of the $\rho_{\text {NIR }}$ and $\rho_{S W I R}$ bands and in this paper we compare five spectral indices which are achieved on Sentinel-2 data as follows: 


\begin{tabular}{cccc}
\hline Acquisition date & Mission & Cloud cover & Fire event \\
\hline $09 / 12 / 2016$ & S-2A & NO & - \\
$29 / 03 / 2017$ & S-2A & NO & - \\
$08 / 04 / 2017$ & S-2A & NO & - \\
$27 / 06 / 2017$ & S-2A & PARTIAL & pre-fire \\
$07 / 07 / 2017$ & S-2A & PARTIAL & post-fire \\
$12 / 07 / 2017$ & S-2B & NO & during-fire \\
$17 / 07 / 2017$ & S-2A & PARTIAL & during-fire \\
$06 / 08 / 2017$ & S-2A & NO & post-fire \\
$16 / 08 / 2017$ & S-2A & NO & - \\
$31 / 08 / 2017$ & S-2B & NO & - \\
$15 / 10 / 2017$ & S-2A & NO & - \\
$04 / 11 / 2017$ & S-2A & NO & - \\
$19 / 12 / 2017$ & S-2B & NO & - \\
$24 / 12 / 2017$ & S-2A & NO & - \\
\hline
\end{tabular}

Table 2. Acquisition dataset from Sentinel-2 mission used for this study.

$$
\begin{gathered}
A F I_{1}=\frac{\rho_{12}}{\rho_{8 A}}+\frac{\rho_{12}}{\rho_{11}}+\alpha \frac{\rho_{8 A}}{\rho_{11}}, \\
A F I_{2}=\frac{\rho_{12}}{\rho_{8 A}}, \\
A F I_{3}=\frac{\rho_{12}}{\rho_{8}}, \\
A F I_{4}=\frac{\rho_{11}}{\rho_{8}}, \\
A F I_{5}=\frac{\rho_{12}}{\rho_{11}}
\end{gathered}
$$

where $\rho_{8}$ and $\rho_{11}$ have been defined in Section 3.1; while $\rho_{8 A}$ is the NIR band centered at the wavelength of $865 \mathrm{~nm}$ and $\rho_{12}$ is the SWIR band, centered at $2190 \mathrm{~nm}$. The $\rho_{8}$ is provided at $10-\mathrm{m}$ spatial resolution, while the other considered bands are provided at 20-m spatial resolution, so the $\rho_{8}$ is resampled at the lower 20-m spatial resolution. It is worthwhile to notice that, since each index refers only to one acquisition date, it is not strictly necessary working on BOA products for this task [16]. Therefore, all of these bands represent the radiance data at TOA, that means the Level-1C. In order to detect an active fire we considered the followed rules:

$$
A F I_{k}>T_{k}
$$

where $T_{k}$ is the threshold for the index $A F I_{k}$ with $k \in\{1,2,3,4,5\}$. 


\subsection{Post-fire analysis}

In order to estimate the extension of a certain burned area, the change detection method is commonly adopted [18,19]. After the selection of a proper spectral index, the technique consists in the computation of such index from Level-2A data [16] acquired before and after the fire event and then in thresholding their difference. Similarly to the active fire case, also in this case the choice of the best index to be use determines the reliability of the technique. In literature there are several spectral indices that can be useful for this purpose. In this work, we used one of the most popular that is the Normalized Differential Vegetation Index (NDVI) and is defined as [20]:

$$
N D V I=\frac{\rho_{N I R}-\rho_{R E D}}{\rho_{N I R}+\rho_{R E D}}
$$

$\rho_{\text {NIR }}$ is even the BOA reflectance value of the NIR band and $\rho_{R E D}$ of the RED band, and on the Sentinel-2 bands as:

$$
N D V I=\frac{\rho_{8}-\rho_{4}}{\rho_{8}+\rho_{4}}
$$

where $\rho_{8}$ has been previously defined; while $\rho_{4}$ is one (Red) of the three Visible bands, centered at $665 \mathrm{~nm}$. Basically, it exploits green leaf scattering in the Near Infra-red wavelength and chlorophyll absorption in the red wavelength. Another index is the Normalized Burning Ratio (NBR) [10,21]. The NBR is defined as [21]:

$$
N B R=\frac{\rho_{N I R}-\rho_{S W I R}}{\rho_{N I R}+\rho_{S W I R}}
$$

$\rho_{\text {NIR }}$ is even the BOA reflectance value of the NIR band and $\rho_{S W I R}$ of the SWIR band, and on the Sentinel-2 bands as:

$$
N B R=\frac{\rho_{8}-\rho_{12}}{\rho_{8}+\rho_{12}}
$$

where $\rho_{8}$ and $\rho_{12}$ have been defined in Section 3.2. In this analysis, we also used the NDWI, above-defined in Section 3.1.

\section{Experimental Results}

\subsection{Classification Metrics}

For the active fire detection and the burned area assessment, the performance of each spectral index used is evaluated in terms of binary classification. To this aim we need to define a ground truth on which computing the classification metrics. For this work, such ground truth (GT) has been generated by hand in GIS environment, using the information derived from the comparison between satellite and high resolution hyperspectral images. Therefore, we compare this GT with the maps obtained thresholding the computed spectral indices. In the specific case, we have considered different thresholds to match the best detection of the active fires and burned areas. In order to evaluate the quality of the retrieved binary maps, we have considered some metrics, usually used for classification tasks [22]:

- Precision $(\mathrm{P})$ is the ratio between the correctly predicted positive observations and the total predicted positive ones;

- Recall (R) is the ratio between the correctly predicted positive observations and all actually positive observations;

- F-measure (F) is the harmonic average of precision and recall.

It is worthwhile to remember that a high $\mathrm{P}$ values correspond to a low false positive rate. In other words, lower false alarm higher Precision. Instead, high $R$ values correspond to a low false negative rate, that means higher $\mathrm{R}$ higher detection rate. Therefore, $\mathrm{F}$ is high when both $\mathrm{P}$ and $\mathrm{R}$ are high; it drastically decreases when one of them falls off. 


\subsection{Pre-fire assessment}

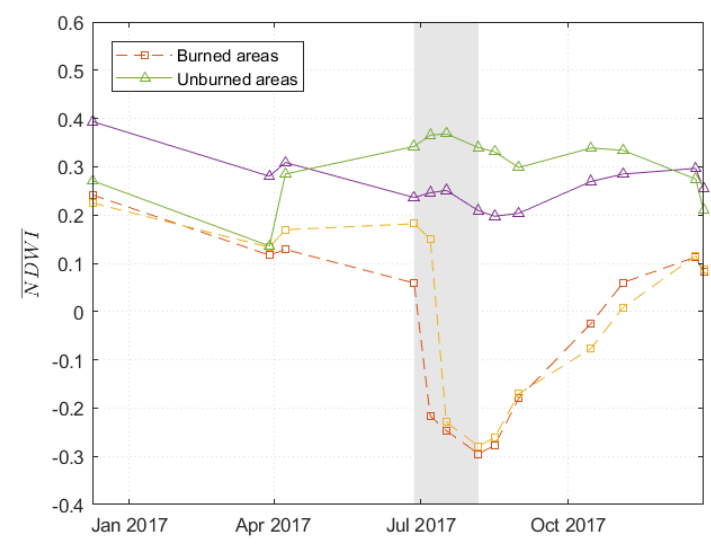

(a)

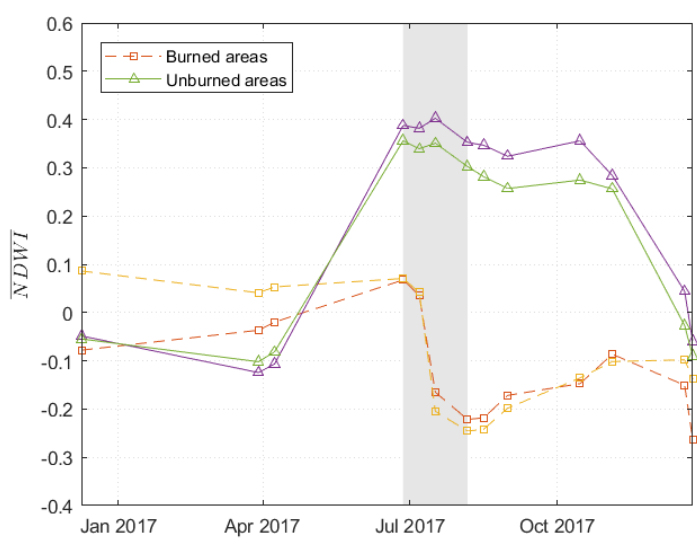

(b)

Figure 4. Time series of the NDWI mean on two AOIs affected by fires (dashed lines) and on other two AOIs that were not (continuous line) for the Vesuvius area (a) and the Sorrento Peninsula (b). Fires period is highlighted in grey.

Thanks to the GT it has been possible to discriminate on the Sentinel-2 data areas affected by fires from those which were not. In order to evaluate the multi-temporal trend of the NDWI, we select several patches from the Vesuvius and the Lattari mountains zones (taking into account the absence of cloud cover). Some samples are shown in Fig. 5. Therefore, for each selected patch and for all the data
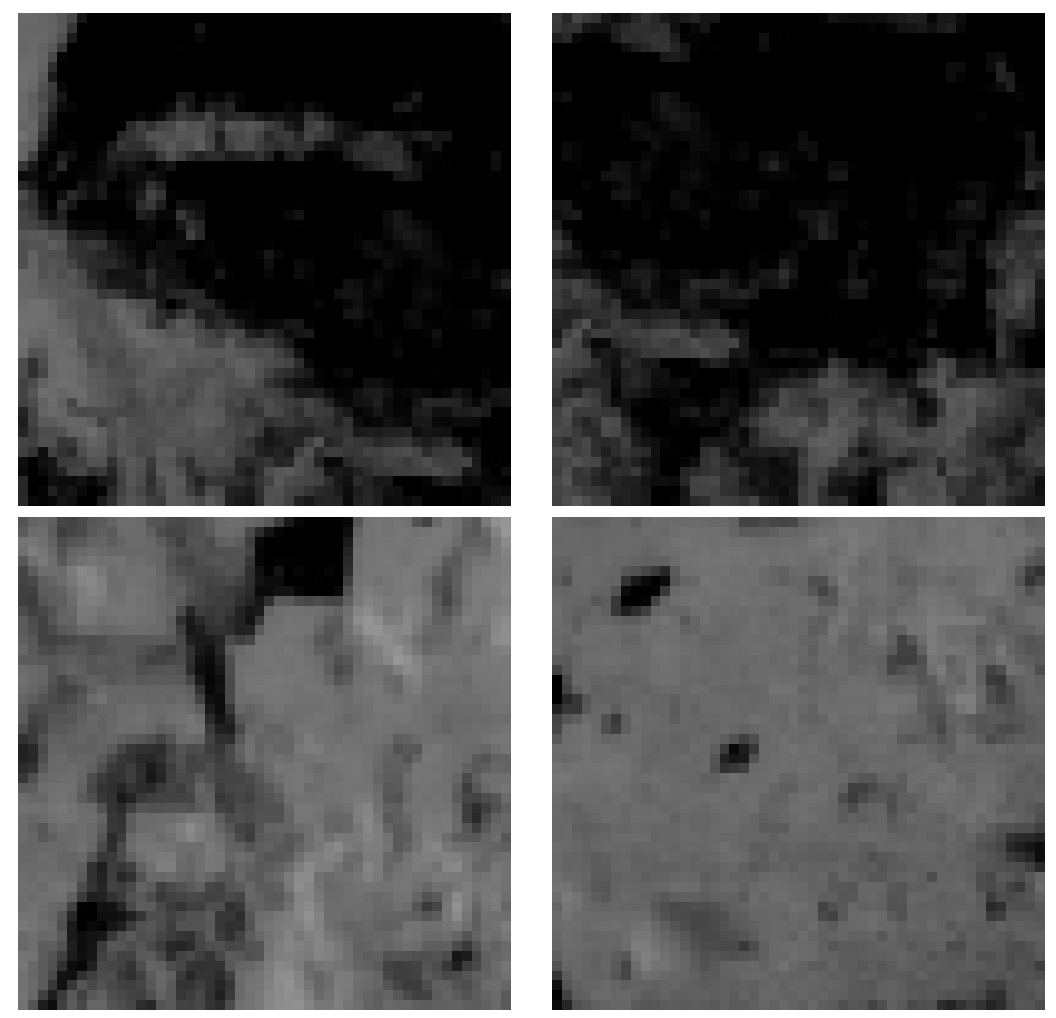

Figure 5. Patches from Vesuvius and Lattari Mountains acquired on June 27th 2017. In the first row, two samples from burned areas (the average values of NDWI in the patches are respectively 0.12 and 0.16 ), and in the second row, two samples from unburned areas (the average values are respectively 0.34 and 0.37$)$. 
frames, the average values of NDWI have been computed, in order to assess the correlation between the fire ignition events and the status of the vegetation. The results are shown in Fig.4. Exploring the graphs we can notice that, after the fire event occurs (at the beginning of July 2017), the NDWI mean value in the burned areas rapidly decreases towards negative values. This is consistent with the fact that lower NDWI value indicates lower water content in the plants, and therefore the presence of a very dry vegetation. Moreover, it is very interesting to focus on the average value of the NDWI before the fire ignition event, in fact in the area affected by fires the average NDWI is lower than the one of areas not affected by fire. In both the analyzed sites this difference seems to be evident, but in the area of the Sorrento peninsula it is much more. In fact, in the first few days of July (before the fire) the NDWI mean value of the burned areas is about four times smaller than that one of the unburned areas. Therefore, this index seems to be a promising tool to identify vegetated areas with higher risk to be affected by fires.

\subsection{During fire assessment}

An active fire is detected by considering the followed rules: $A F I_{k}>T_{k}$, where $k \in\{1,2,3,4,5\}$. The performance is computed in terms of the metrics described in Sec. 4.1 and is reported in Table 3. Moreover, in order to evaluate the threshold stability, the technique has been applied on both sites under investigation, in fact in Table 3 it is noticed that we have obtained a different threshold for the two considered areas of interest. In particular, the AFIs have been computed on Sentinel-2 data acquired on 12th and 17th July 2017 for the Vesuvius and Sorrento areas, respectively. As we can appreciate in Table 3, the $A F I_{1}$ and $A F I_{5}$ show the best performance in terms of F-measure for both sites. In particular, the presented result for $A F I_{1}$ has been obtained with $\alpha=0.5$, as reported in [17]. On the opposite, $A F I_{4}$ presents the worst F-measure for both the target sites. This is due to the very low recall value that means a low detection rate. Furthermore this index has been computed after the down-scaling of the $\rho_{8}$ band to the 20-m spatial resolution and it can determine a general worsening in the performance. This aspect can have a greater impact where the active fires becomes thinner. Indeed, observing the Vesuvius area in Fig. 6, we can see that the upper-thinner line of fire is almost absent in the $A F I_{2}$ and $A F I_{3}$, and completely absent in $A F I_{4}$ map. These results allow us to conclude that resampling of the $\rho_{8}$ band degrades performances, and further $\rho_{12}$ band is more effective than $\rho_{11}$ when is considered in a ratio with $\rho_{\text {NIR }}$. However, among the indices with worst performance, $A F I_{2}$ and $A I_{3}$ have the better one in terms of precision (especially for the Vesuvius area), that means low false alarm rate. This aspect can be easily verified observing the corresponding maps in Fig.6. Moreover, from a visual inspection we can observe that the boundaries (especially for very thin active fires) are more evident considering the indices which involve $\rho_{12}$ and $\rho_{11}$ bands than those using only $\rho_{12}$ and $\rho_{8}$, or $\rho_{8 A}$ bands. Similar considerations can be carried out for the Sorrento area. It can be

\begin{tabular}{|c|c|c|c|c|}
\hline & Precision & Recall & F-measure & Threshold \\
\hline \multirow{2}{*}{$A F I_{1}$} & 0.8206 & 0.5389 & 0.6505 & 0.14 \\
\hline & 0.7962 & 0.6327 & 0.7051 & 0.27 \\
\hline \multirow{2}{*}{$A F I_{2}$} & 0.9694 & 0.3340 & 0.4968 & 0.11 \\
\hline & 0.8584 & 0.4958 & 0.6286 & 0.15 \\
\hline \multirow{2}{*}{$A F I_{3}$} & 0.9649 & 0.3416 & 0.5045 & 0.10 \\
\hline & 0.7434 & 0.5609 & 0.6394 & 0.12 \\
\hline \multirow{2}{*}{$A F I_{4}$} & 0.4652 & 0.1308 & 0.2042 & 0.14 \\
\hline & 0.6189 & 0.2738 & 0.3796 & 0.17 \\
\hline \multirow{2}{*}{$A F I_{5}$} & 0.9265 & 0.5220 & 0.6678 & 0.19 \\
\hline & 0.7477 & 0.6878 & 0.7165 & 0.22 \\
\hline
\end{tabular}

Table 3. Table of AFIs performance on the study areas.

finally noted in Table 3 that the optimal threshold values are very similar in the two considered scenes 


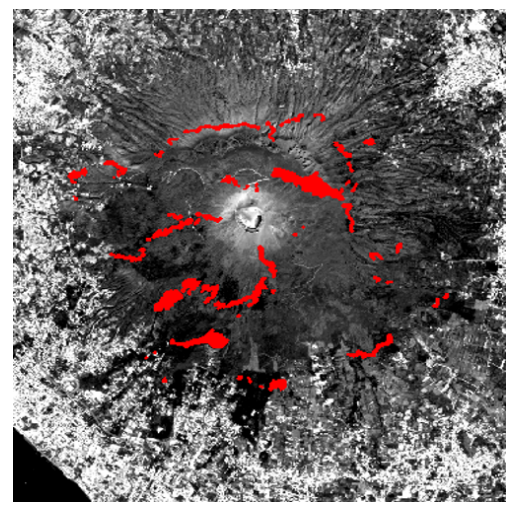

GT

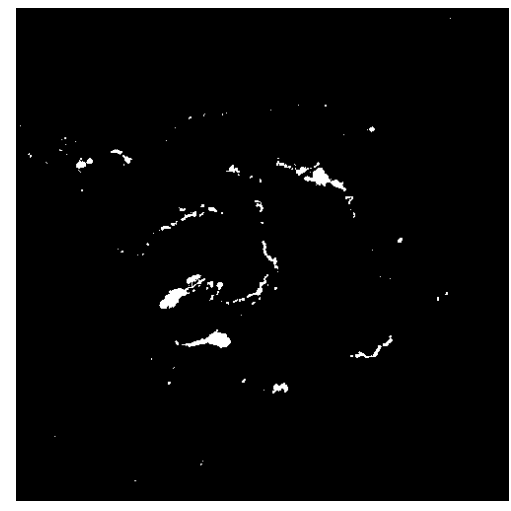

$A F I_{3}$ map

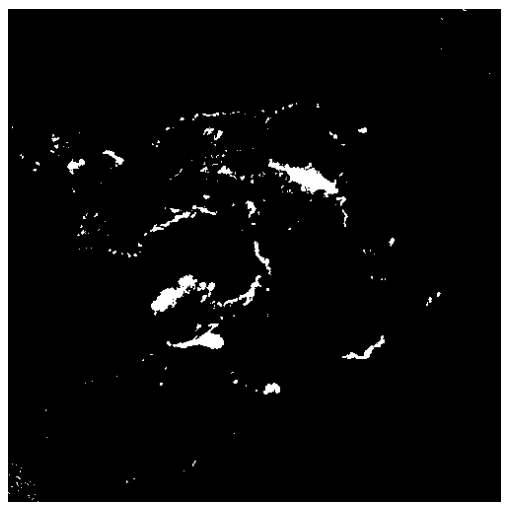

$A F I_{1}$ map

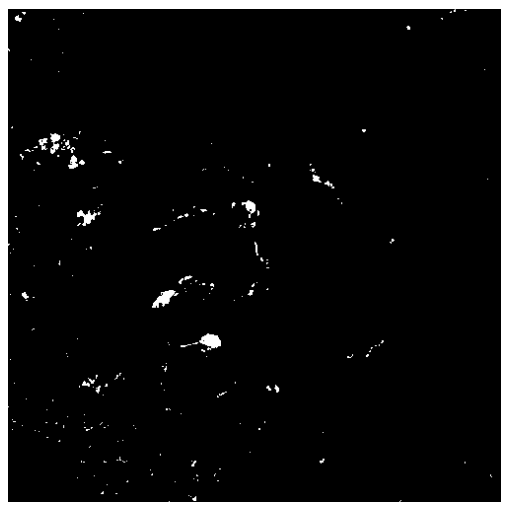

$A F I_{4}$ map

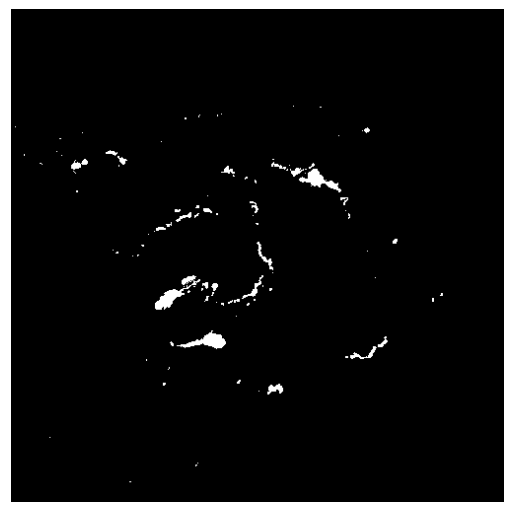

$A F I_{2}$ map

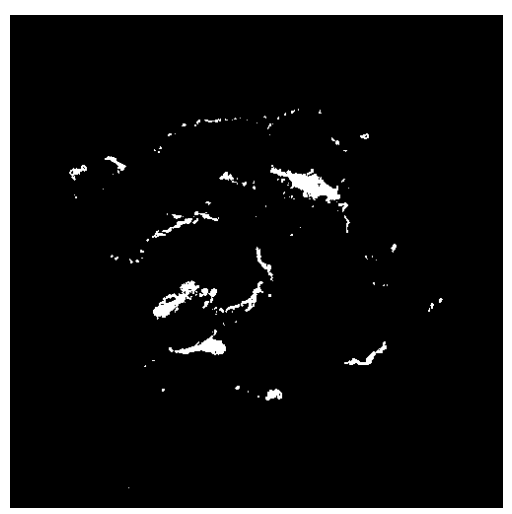

$A F I_{5}$ map

Figure 6. Ground truth used (up-left corner) and the AFIs maps obtained for the active fire detecion task on the Vesuvius area.

for all the indices, with the exception of $A F I_{1}$. Indeed, the threshold value of the $A F I_{1}$ is very different moving from one case to the other. This is probably due to the $\alpha$ coefficient which has to be properly tuned when considering different areas with different features. This instability does not occur for $A F I_{5}$, and in general for AFIs that involve only two bands, which show more stable threshold values.

\subsection{Post-fire assessment}

For the numerical assessment we have compared all the indices mentioned in Sec.3.3 (included the NDWI, defined in Section 3.1) in terms of binary classification.

\begin{tabular}{|c|c|c|c|c|}
\hline & Precision & Recall & F-measure & Threshold \\
\hline \multirow{2}{*}{ NDWI } & 0.8657 & 0.6358 & 0.7332 & 0.05 \\
\hline & 0.9317 & 0.9057 & 0.9185 & 0.07 \\
\hline \multirow{2}{*}{ NDVI } & 0.8893 & 0.8226 & 0.8546 & 0.18 \\
\hline & 0.9206 & 0.8079 & 0.8606 & 0.06 \\
\hline \multirow{2}{*}{ NBR } & 0.9500 & 0.8073 & 0.8728 & 0.04 \\
\hline & 0.9345 & 0.9253 & 0.9299 & 0.08 \\
\hline
\end{tabular}

Table 4. Table of the performance of the proposed indices for the burned area mapping task on the study areas.

The proposed analysis has been then performed on both the subject areas of this work. In particular, for the Vesuvius volcano area we assess the change detection related to the fire event of 


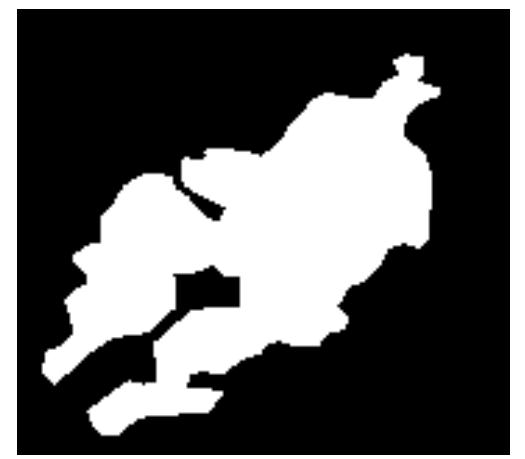

GT

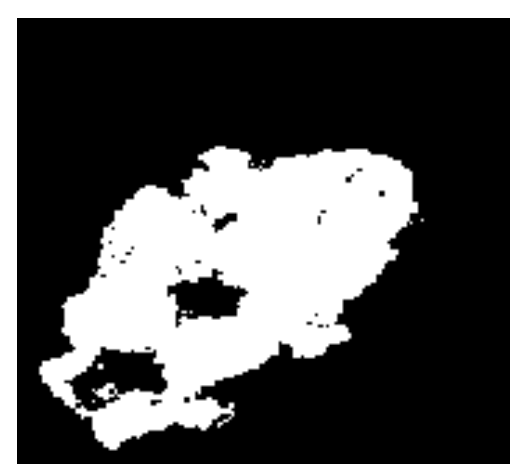

NDVI map

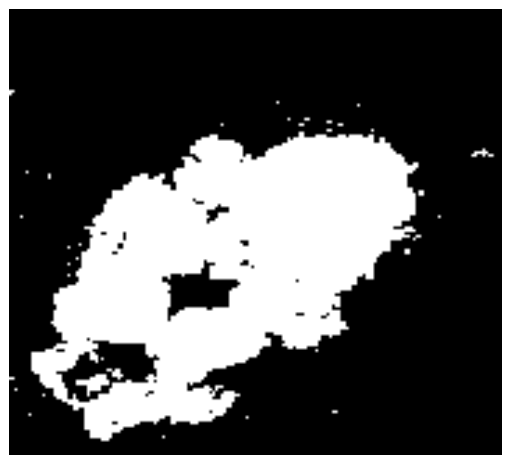

NDWI map

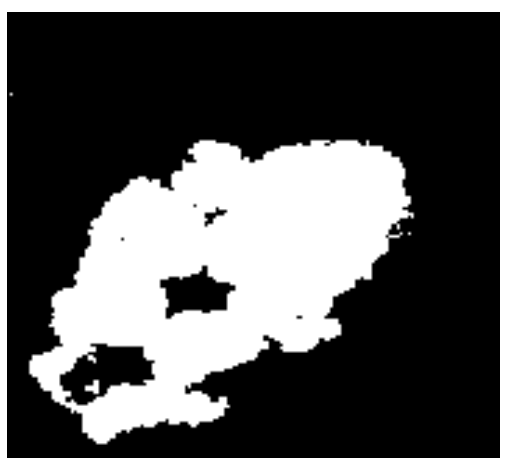

NBR map

Figure 7. Ground truth used (up-left corner) and the maps obtained for the burned area mapping task.

the July, 5th 2017, and thus we have used the Sentinel-2 data acquired on June 27th and July 7th 2017 as pre-fire and post-fire images, respectively. While the acquisition of the July 17th 2017 has been taken into account as post-fire image, for the Sorrento peninsula. The numerical results are shown in Table 4 for both the study areas. The NBR index provides the better performance for both the target sites, even if in the Vesuvius area the NDVI shows a higher value for the recall metric. However the F-measure values of the NDVI and NDWI do not appear extremely worse with respect to the NBR one, especially for the Sorrento area. Although this could suggest to use one of them indifferently, we have to consider, as in the active fire detection task, the threshold stability that also is a crucial aspect in this case. Therefore, observing the last column of the Table 4, it can be noticed that the threshold values of the NBR index and the NDWI are more stable compared to the NDVI one. Moreover, on the same target site, the NBR and the NDWI present threshold values very similar, but the former with a better F-measure. For these reasons, the use of the NBR is suggested. From a visual point of view (see Fig. 7), it is possible to appreciate the good quality of the map obtained on a small area of the Vesuvius park using the NBR index. Finally, about the spatial resolution, it is worthwhile to remember that NBR and NDWI are defined at 20-m spatial resolution; while the NDVI involves 10-m resolution bands, but in Fig. 7 it is displayed an its version scaled at $20-\mathrm{m}$ resolution just for a better presentation without any lost meaning on the reported considerations.

\section{Conclusions and future developments}

In conclusion, in this article we highlight how satellite remote sensing products can make a huge contribution to protecting the environment from the forest fires threat. In particular, the use of the NDWI to monitor the water content of the vegetation areas, in order to perform a fires risk prevention task, has been tested. The encouraging results obtained by a multi-temporal analysis of the NDWI allow us to further investigate and explore new ways to develop useful remote sensing techniques for the ambitious forest fire prevention purpose. Regarding the during-fire assessment, standard active fire detection technique based on the definition of spectral indices have been presented and compared. In 
general, the $A F I_{5}$, composed by the SWIR bands, gives better results in terms of the considered metrics. Moreover, the AFIs defined as the ratio between two bands present more stable and reliable threshold values with respect to the $A F I_{1}$ defined as the linear combination of three specific ratios. Then, based on change detection analysis, a comparison between three indices (NBR, NDVI and NDWI) has been performed in order to ensure accurate burned areas detection. The presented analysis has highlighted that the detection capability and the threshold stability are not negligible aspects in the choice of which index has to be used to accomplish this task. An interesting possible future development of this study is the integration of presented techniques, based on satellite Sentinel-2 data, with detection methods based on airborne extremely high resolution thermal sensors. An example of such a sensor is the last-generation ITRES TABI-1800 thermal sensor (see Table 5 for its technical specifications) mounted on the Tecnam P2006T special mission aircraft (Fig. 8) of BENECON S.C.aR.L. In fact, guided by the large-scale Sentinel-2 fire maps, the aircraft can be sent over critical areas to detect very small fire fronts.

\begin{tabular}{lc}
\hline Parameter & Description \\
\hline Sensor type & TIR Pushframe sensor \\
Spectral range & $3.7-4.8 \mu \mathrm{m}$ \\
\# Spectral channels & 1 \\
\# Across-Track pixels & 1800 \\
Total Field of View & 40 degrees \\
IFOV (+/- 10\%) & 0.405 mrad \\
Spatial resolution & $10-125 \mathrm{~cm}$ \\
\hline
\end{tabular}

Table 5. ITRES TABI-1800 specifications.

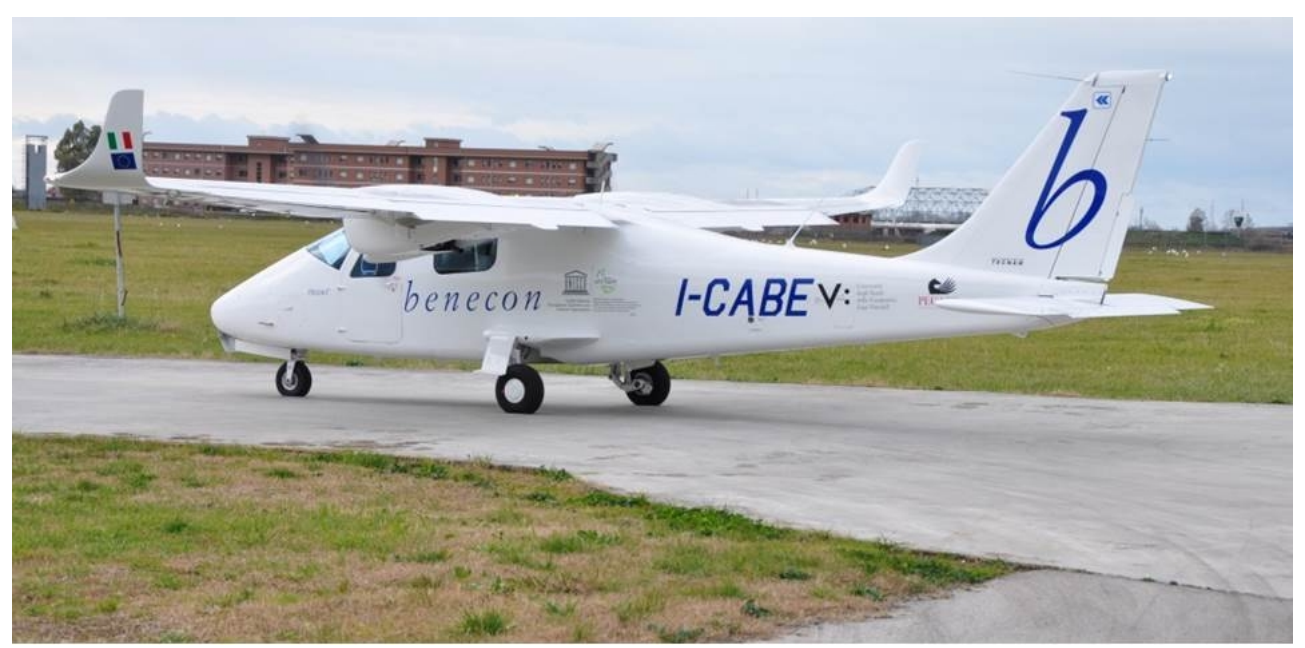

Figure 8. The Tecnam P2006T aircraft owned by Benecon S.C.aR.L.

Author Contributions: Conceptualization, D.A.G.D., R.P. and D.R.; methodology, M.G., D.A.G.D., and A.I.; software, M.G., and D.A.G.D.; validation, D.A.G.D., and M.G.; data curation, M.G., D.A.G.D, and R.P.; writing-original draft preparation, D.A.G.D.; writing—review and editing, M.G., A.I., R.P., and G.R.; supervision, D.R., C.G., and A.I.; project administration, C.G. and D.R.

Funding: This research received no external funding.

Conflicts of Interest: The authors declare no conflict of interest. 


\section{References}

1. J. Kaufman, Y.; O. Justice, C.; P. Flynn, L.; D. Kendall, J.; Prins, E.; Giglio, L.; Ward, D.; Paul Mensel, W.; Setzer, A. Potential global fire monitoring from EOS-MODIS. J. Geophys. Res. 1998, 103. doi:10.1029/98JD01644.

2. Schroeder, W.; Oliva, P.; Giglio, L.; Csiszar, I. The New VIIRS $375 \mathrm{~m}$ active fire detection data product: Algorithm description and initial assessment. Remote Sensing of Environment 2014, 143, 85-96. doi:10.1016/j.rse.2013.12.008.

3. Gao, B.C. NDWI?A Normalized Difference Water Index for Remote Sensing of Vegetation Liquid Water from Space. Remote Sensing of Environment 1996, 58, 257-266. doi:10.1016/S0034-4257(96)00067-3.

4. Huete, A.R.; Liu, H.; van Leeuwen, W.J. The use of vegetation indices in forested regions: issues of linearity and saturation. IGARSS'97. 1997 IEEE International Geoscience and Remote Sensing Symposium Proceedings. Remote Sensing-A Scientific Vision for Sustainable Development. IEEE, 1997, Vol. 4, pp. 1966-1968.

5. Semeraro, T.; Vacchiano, G.; Aretano, R.; Ascoli, D. Application of vegetation index time series to value fire effect on primary production in a Southern European rare wetland. Ecological Engineering 2019, 134, 9-17.

6. Lentile, L.; Holden, Z.; M. S. Smith, A.; Falkowski, M.; Hudak, A.; A Morgan, P.; Lewis, S.; Gessler, P.; Benson, N. Remote sensing techniques to assess active fire characteristics and post-fire effects. International Journal of Wildland Fire 2006, 15. doi:10.1071/WF05097.

7. Schroeder, W.; Oliva, P.; Giglio, L.; Quayle, B.; Lorenz, E.; Morelli, F. Active fire detection using Landsat-8/OLI data. Remote Sensing of Environment 2015, 185. doi:10.1016/j.rse.2015.08.032.

8. Barducci, A.; Guzzi, D.; Marcoionni, P.; Pippi, I. Infrared detection of active fires and burnt areas: theory and observations. Infrared physics \& technology 2002, 43, 119-125.

9. Gargiulo, M.; Dell'Aglio, D.; Iodice, A.; Riccio, D.; Ruello, G. A CNN-Based Super-Resolution Technique for Active Fire Detection on Sentinel-2 Data. arXiv 2019. arXiv preprint arXiv:1906.10413.

10. Huang, H.; Roy, D.; Boschetti, L.; Zhang, H.; Yan, L.; Kumar, S.; Gomez-Dans, J.; Li, J. Separability Analysis of Sentinel-2A Multi-Spectral Instrument (MSI) Data for Burned Area Discrimination. Remote Sensing 2016, 8. doi:10.3390/rs8100873.

11. San Miguel Ayanz, J.; Barbosa, P.; Schmuck, G.; Liberta, G.; Schulte, E.; Gitas, I. Towards a coherent forest fire information system in Europe: the European Forest Fire Information System (EFFIS) 2002.

12. McFerren, G.; Frost, P. Southern african advanced fire information system 2009.

13. Drusch, M.; Del Bello, U.; Carlier, S.; Colin, O.; Fernandez, V.; Gascon, F.; Hoersch, B.; Isola, C.; Laberinti, P.; Martimort, P.; others. Sentinel-2: ESA's optical high-resolution mission for GMES operational services. Remote sensing of Environment 2012, 120, 25-36.

14. Immitzer, M.; Vuolo, F.; Atzberger, C. First experience with Sentinel-2 data for crop and tree species classifications in central Europe. Remote Sensing 2016, 8, 166.

15. Dell'Aglio, D.; Gargiulo, M.; Iodice, A.; Riccio, D.; Ruello, G. Active Fire Detection in Multispectral Super-Resolved Sentinel-2 Images by Means of Sam-Based Approach. 2019 IEEE 5th International forum on Research and Technology for Society and Industry (RTSI). IEEE, 2019, pp. 124-127.

16. Song, C.; Woodcock, C.E.; Seto, K.C.; Lenney, M.P.; Macomber, S.A. Classification and change detection using Landsat TM data: when and how to correct atmospheric effects? Remote sensing of Environment 2001, 75, 230-244.

17. Cicala, L.; Angelino, C.; Fiscante, N.; Ullo, S. Landsat-8 and Sentinel-2 for fire monitoring at a local scale: A case study on Vesuvius. 2018 IEEE International Conference on Environmental Engineering (EE). IEEE, 2018, pp. 1-6.

18. Singh, A. Review article digital change detection techniques using remotely-sensed data. International journal of remote sensing 1989, 10, 989-1003.

19. Chuvieco, E.; Martin, M.P.; Palacios, A. Assessment of different spectral indices in the red-near-infrared spectral domain for burned land discrimination. International Journal of Remote Sensing 2002, 23, 5103-5110.

20. Fernández, A.; Illera, P.; Casanova, J.L. Automatic mapping of surfaces affected by forest fires in Spain using AVHRR NDVI composite image data. Remote sensing of environment 1997, 60, 153-162.

21. Miller, J.D.; Thode, A.E. Quantifying burn severity in a heterogeneous landscape with a relative version of the delta Normalized Burn Ratio (dNBR). Remote Sensing of Environment 2007, 109, 66-80. 
22. Patil, T.R.; Sherekar, S.; others. Performance analysis of Naive Bayes and J48 classification algorithm for data classification. International journal of computer science and applications 2013, 6, 256-261.

Sample Availability: Samples of the compounds ...... are available from the authors. 